\title{
Complex coronary anatomy in coronary artery bypass graft surgery: Impact of complex coronary anatomy in modern bypass surgery? Lessons learned from the SYNTAX trial after two years
}

\author{
Friedrich W. Mohr, MD, PhD, ${ }^{a}$ Ardawan J. Rastan, MD, PhD, ${ }^{a}$ Patrick W. Serruys, MD, PhD, ${ }^{\mathrm{b}}$ \\ A. Pieter Kappetein, MD, PhD, ${ }^{\mathrm{b}}$ David R. Holmes, MD, PhD, ${ }^{\mathrm{c}}$ Jose L. Pomar, MD, PhD, ${ }^{\mathrm{d}}$ \\ Stephen Westaby, MD, PhD, ${ }^{\mathrm{e}}$ Katrin Leadley, MD, ${ }^{\mathrm{f}}$ Keith D. Dawkins, MD, ${ }^{\mathrm{f}}$ and \\ Michael J. Mack, MD, $\mathrm{PhD}^{\mathrm{g}}$
}

Objective: SYNTAX study compares outcomes of coronary artery bypass grafting with percutaneous coronary intervention in patients with 3-vessel and/or left main disease. Complexity of coronary artery disease was quantified by the SYNTAX score, which combines anatomic characteristics of each significant lesion. This study aims to clarify whether SYNTAX score affects the outcome of bypass grafting as defined by major adverse cerebrovascular and cardiac events (MACCE) and its components over a 2-year follow-up period.

\begin{abstract}
Methods: Of the 3075 patients enrolled in SYNTAX, 1541 underwent coronary artery bypass grafting (897 randomized controlled trial patients, and 644 registry patients). All patients undergoing bypass grafting were stratified according to their SYNTAX score into 3 tertiles: low (0-22), intermediate (22-32), and high $(\geq 33)$ complexity. Clinical outcomes up to 2 years after allocation were determined for each group and further risk factor analysis was performed.
\end{abstract}

Results: Registry patients had more complex disease than those in the randomized controlled trial (SYNTAX score: registry $37.8 \pm 13.3$ vs randomized $29.1 \pm 11.4 ; P<.001$ ). At 30 days, overall coronary bypass mortality was $0.9 \%$ (registry $0.6 \%$ vs randomized $1.2 \%$ ). MACCE rate at 30 days was $4.4 \%$ (registry $3.4 \%$ vs randomized $5.2 \%$ ).

SYNTAX score did not significantly affect overall 2-year MACCE rate of $15.6 \%$ for low, $14.3 \%$ for medium, and $15.4 \%$ for high SYNTAX scores. Compared with randomized patients, registry patients had a lower rate of overall MACCE rate (registry $13.0 \%$ vs randomized $16.7 \% ; P=.046)$ and repeat revascularization $(4.7 \%$ vs $8.6 \% ; P=.003)$, whereas other event rates were comparable. Risk factor analysis revealed left main disease $(P=.049)$ and incomplete revascularization $(P=.005)$ as predictive for adverse 2-year outcomes.

Conclusions: The outcome of coronary artery bypass grafting was excellent and independent from the SYNTAX score. Incomplete revascularization rather than degree of coronary complexity adversely affects late outcomes of coronary bypass. (J Thorac Cardiovasc Surg 2011;141:130-40)

\footnotetext{
From the Department of Cardiac Surgery, ${ }^{a}$ Heart Center, University of Leipzig, Germany; Erasmus University Medical Center, ${ }^{\mathrm{b}}$ Rotterdam, The Netherlands; Mayo Clinic, ${ }^{\mathrm{c}}$ Rochester, Minn; Department of Cardiovascular Surgery, ${ }^{\mathrm{d}}$ Hospital Clinico de Barcelona, Spain; John Radcliffe Hospital, ${ }^{\mathrm{e}}$ Oxford, United Kingdom; Boston Scientific Corporation, ${ }^{\mathrm{f}}$ Marlborough, Mass; and Medical City Hospital, ${ }^{\mathrm{g}}$ Dallas, Tex.

Supported by Boston Scientific Corporation Clinical Trial Registration Information: NCT00114972.

Disclosures: Friedrich W. Mohr and Patrick W. Serruys were the SYNTAX study principal investigators and A. Pieter Kappetein was co-principal investigator. Dr. Kappetein reports receiving consulting and lecture fees from Boston Scientific. Katrin Leadley and Keith Dawkins are full-time employees of Boston Scientific and receive a salary and hold equity in the company. All other authors were site investigators and have no other conflicts of interest. This study was supported by Boston Scientific, but the company was not involved in the decision to publish the results of the study. All authors have participated in the interpretation of the data, drafting and review of this manuscript, and have approved its submission.

Read at the 90th Annual Meeting of The American Association for Thoracic Surgery, Toronto, Ontario, Canada, May 1-5, 2010.

Received for publication May 11, 2010; revisions received July 4, 2010; accepted for publication July 19, 2010.

Address for reprints: Friedrich W Mohr, MD, PhD, University Leipzig, Department of Cardiac Surgery, Struempellstr 39, 04289 Leipzig, Germany (E-mail: mohrf@ medizin.uni-leipzig.de).

0022-5223/\$36.00

Copyright (C) 2011 by The American Association for Thoracic Surgery doi:10.1016/j.jtcvs.2010.07.094
}

Coronary artery bypass grafting (CABG) rather than percutaneous coronary intervention (PCI) is recommended to be the primary revascularization strategy for patients with stable angina who have coronary triple vessel disease (3-VD) or left main (LM) disease. ${ }^{1-4}$ This recommendation is based on results of several randomized controlled trials, metaanalyses and registry data comparing CABG surgery with bare metal stent as well as contemporary PCI technologies. ${ }^{5-8}$ Increasing use of drug-eluting stents and greater operator experience has resulted in PCI being performed in patients with increasingly complex coronary pathologic conditions and class I surgical indications. ${ }^{9}$ Studies comparing PCI with drug-eluting stents to CABG for prognostic indications have generally been limited to nonrandomized studies thus far and have demonstrated significant advantages of CABG over PCI for repeat revascularization. However, such studies have failed to demonstrate survival benefit for CABG, probably because of inadequately powered study designs. ${ }^{10-12}$ Nevertheless, surgeons are 


Abbreviations and Acronyms
$\begin{aligned} \text { CABG } & \text { coronary artery bypass grafting } \\ \text { CVA } & =\text { cerebrovascular accident } \\ \text { ITA } & =\text { internal thoracic artery } \\ \text { LAD } & \text { left anterior descending coronary } \\ & \text { artery } \\ \text { LM } & =\text { left main } \\ \text { MACCE }= & \text { major adverse cardiac and } \\ & \text { cerebrovascular event } \\ \text { MI }= & \text { myocardial infarction } \\ \text { OUS } & \text { outside the United States } \\ \text { PCI } & =\text { percutaneous coronary intervention } \\ \text { SYNTAX }= & \text { SYNergy between PCI with TAXUS } \\ & \text { and Cardiac Surgery } \\ \text { US } & =\text { United States } \\ \text { VD } & =\text { vessel disease }\end{aligned}$

confronted with the widespread use of PCI in these patients despite conflicting support from evidence-based medicine.

The SYNTAX (SYNergy between PCI with TAXUS and Cardiac Surgery) trial thus assessed for the first time the optimal revascularization strategy in 1800 randomized patients with de novo 3-VD or LM disease comparing contemporary bypass surgery and PCI with drug-eluting stents by using a noninferiority study design. ${ }^{13}$ Patients ineligible for PCI treatment were entered into a parallel, nested CABG registry, and patients deemed ineligible for CABG were treated with PCI and data integrated in the PCI registry.

As recently demonstrated, the primary study end point of major adverse cardiac and cerebrovascular event rate (MACCE) at 1 year was significantly lower for CABG $(12.4 \%)$ than for PCI $(17.8 \%)$ patients. ${ }^{14}$

A secondary objective of the SYNTAX trial was to analyze the complexity of the coronary artery disease by using a newly developed SYNTAX score that was derived from various pre-existing angiographic anatomic classifications. ${ }^{15}$ A subgroup analysis of the randomized SYNTAX study population revealed significant evidence of worse PCI results in patients with increasingly complex coronary anatomy. ${ }^{14}$ This observation was recently confirmed by retrospectively applying the SYNTAX score to other PCI patient groups, that is, ARTS II and LM disease. ${ }^{16,17}$ In contrast, surgical results were less adversely affected by coronary complexity in the randomized CABG population. Moreover; there was some evidence of higher 1 -year adverse event rates in the low SYNTAX score group, but this was not observed in the CABG registry at 1 year's follow-up. ${ }^{14,17,18}$

The aim of this analysis was to assess the impact of coronary complexity on surgical outcomes up to 2 years post- operatively and to identify potential clinical and coronary risk factors for adverse CABG results by analyzing the entire SYNTAX CABG population (randomized and registry), which can be considered as representative for contemporary bypass surgery.

\section{METHODS \\ Study Design and Allocation}

Between March 2005 and May 2007, a total 85 sites in Europe (17 countries, 62 sites) and the United States (US, 23 sites) enrolled patients in the SYNTAX study. The institutional review board at each site approved the protocol, and all patients provided written informed consent. The protocol and consent forms were consistent with the Food and Drug Administration's Guidance for Industry E6 Good Clinical Practice, the Declaration of Helsinki, the International Conference on Harmonisation, and all local regulations, as appropriate.

In contrast to previous comparative studies, patients with significant cardiac diseases (eg, poor ejection fraction, unstable angina) and severe comorbidities (eg, renal dysfunction, carotid artery disease) were not excluded from SYNTAX, thus allowing the study to better represent real clinical practice. The SYNTAX "all-comers" study design and patient selection have been previously described in detail. ${ }^{13,14}$ In brief, patients with de novo 3-VD and/or LM disease were screened for study suitability by a local Heart Team that was composed of an interventional cardiologist, a cardiac surgeon, and the study coordinator. If the consensus decision was that comparably complete revascularization could be achieved by either PCI or CABG therapy, the patient was randomized to one of the treatment options. A total of 903 patients were assigned to PCI using TAXUS Express paclitaxel-eluting stents (Boston Scientific, Natick, Mass) and 897 patients were assigned to CABG surgery. ${ }^{13}$ The primary study end point for the randomized cohort was the overall MACCE rate at 12 months' follow-up, but patients were followed up to 5 years after allocation. The SYNTAX study also included two nested registries, one for PCI-ineligible patients (CABG registry) and one for CABG-ineligible patients (PCI registry). A total of 1079 patients were allocated to the CABG registry. Reasons not to randomize these patients were predominantly complex coronary anatomy $(70.9 \%)$ and potentially untreatable coronary chronic total occlusion $(22.0 \%)$. Of these patients, 649 were randomly assigned to 5-year follow-up, of whom 5 had to be excluded initially because of no revascularization or medical treatment only. During 2-year follow-up, 12 patients were lost to follow-up or withdrew study consent (follow-up 98.1\% complete). From 897 randomized CABG patients, 51 withdrew study participation and 10 were lost to follow-up, leading to a 2-year follow-up rate of $93.2 \%$ (836 patients). Therefore, a total of $1541 \mathrm{SYN}$ TAX CABG patients were assigned to 5 years' follow-up and analyzed for the current study (897 randomized and 644 registry patients). Overall 2-year follow-up was available on 1468 CABG patients, corresponding to a follow-up rate of $95.3 \%$.

\section{Assessment of Coronary Complexity}

All preoperative diagnostic angiograms were reviewed by an independent core laboratory blinded to treatment assignment (Cardialysis BV, Rotterdam, the Netherlands). Diagnostic angiograms were scored according to the SYNTAX score algorithm. The SYNTAX score is a comprehensive anatomic assessment of the coronary disease derived from various preexisting anatomic classifications. In brief, each coronary lesion producing $50 \%$ luminal obstruction or greater in vessels $1.5 \mathrm{~mm}$ or larger is defined on the basis of the modified American Heart Association coronary tree segment classification and separately scored regarding segment importance, bifurcation or trifurcation, aortic ostial localization, chronic total occlusion, vessel tortuosity, lesion length, calcification, and thrombus formation. Finally, the score of each lesion is added to obtain a patient-specific raw 
SYNTAX score. ${ }^{15}$ Thus, higher SYNTAX scores are indicative of more complex coronary disease. ${ }^{16}$

\section{CABG Surgery}

All patients were treated with the intention of complete revascularization, defined as bypassing all lesions with $50 \%$ or greater stenosis in vessel diameters of more than $1.5 \mathrm{~mm}$ as identified by the Heart Team. CABG technique was performed according to the surgeon's preference and local clinical standards. It was recommended to revascularize the left anterior descending artery (LAD) and the circumflex branches by using either the left or right internal thoracic artery (ITA), when technically feasible, and the remaining vessels with either another arterial conduit or a saphenous vein in a configuration deemed appropriate by the surgeon. In patients less than 70 years of age, complete arterial revascularization was recommended. It was also left to the surgeon's preference to operate with or without cardiopulmonary bypass and, in case of cardioplegic arrest, to use blood or crystalloid cardioplegia. Anesthesia management, including the use of aprotinin and postoperative care, was also performed according to local clinical standards, as was the postoperative medication regimen. Aspirin was prescribed indefinitely in all randomized CABG patients only.

\section{End Points}

The primary clinical end point was the composite of all MACCEs more than 12 months after allocation. MACCE was defined as all-cause death, cerebrovascular accident (CVA), myocardial infarction (MI), and repeat revascularization. CVA was defined as a focal, central neurologic deficit lasting more than 72 hours that resulted in irreversible brain damage or body impairment. The definition of MI was based on previous studies. ${ }^{10}$ Safety end point was a combination from death, CVA, and MI. Repeat revascularization was defined as any postoperative coronary intervention. Complete revascularization was based on the anatomic (ie, functional) definition by bypassing all eligible lesions identified during the Heart Team conference. An independent Clinical Events Committee including cardiologists, cardiac surgeons, and a neurologist reviewed all primary clinical end points. ${ }^{14}$

\begin{abstract}
Statistical Analysis
Analyses were conducted using SAS system software, version 8.0 or higher (SAS Institute, Inc, Cary, NC), and included patients randomized to $\mathrm{CABG}$ (intent-to-treat) or who underwent $\mathrm{CABG}$ as part of the CABG registry and were allocated to 5-year follow-up. Continuous variables were estimated as mean \pm standard deviation, and randomized and registry patients were compared with the Student $t$ test. Discrete variables were reported as counts and percentages, and differences between randomized and registry patients were assessed by the $\chi^{2}$ or Fisher's exact test. Cumulative event-free rates were estimated by Kaplan-Meier methods with differences between groups compared with the log-rank test. Additionally, patients were divided into groups on the basis of tertiles of the baseline SYNTAX score (low $\leq 22$, intermediate 23-32, and high $\geq 33$ ), and groups were compared in a freedom-from-event manner with a log-rank test. Univariate and multivariate logistic regression models including preoperative and intraoperative variables were used to identify potential predictors of 2-year MACCE. They were expressed as odds ratio $\pm 95 \%$ confidence interval. The multivariate models were created by stepwise selection with an entry and exit criterion for each candidate of $P<.10$, beginning with variables with a $P$ value $<.10$ in the univariate analysis.
\end{abstract}

\section{RESULTS}

\section{Patient Population and Surgical Treatment}

Baseline extracardiac and cardiac characteristics, as well as variables regarding coronary complexity of all
CABG patients, were depicted in Table 1. Baseline patient demographics, clinical characteristics, and operative risk scores of the randomized and registry CABG patients were comparable, with the exceptions of more carotid artery disease, less severe angina, higher operative priority, and lower ejection fraction in the registry population. As expected, major differences between the groups were found concerning the complexity of coronary artery disease indicated by a SYNTAX score of $37.8 \% \pm 13.3 \%$ for registry and $29.1 \% \pm 11.4 \%$ for randomized patients $(P<.001)$. In more detail, predictors of being assigned to the CABG registry were the total number of lesions, the presence of LM disease, especially in combination with 2-VD and 3-VD, chronic vessel occlusion, bifurcation lesions, lesion length greater than $20 \mathrm{~mm}$, and heavy lesion calcification (Table 1).

CABG was performed by conventional techniques in the vast majority $(80.9 \%)$ of patients using predominantly blood cardioplegic arrest (Table 2). A minority of $16.6 \%$ received off-pump bypass surgery. Despite a mean age of 65 years, only $31.5 \%$ of the patients received more than 1 arterial graft. Double ITA was used in $22.7 \%$ and a radial artery in $12.8 \%$, leading to complete arterial revascularization in only $15.6 \%$ of patients. There was a significantly higher rate of bilateral ITA use and complex graft constructions in the randomized cohort. CABG registry patients received a significant higher number of distal anastomoses per patient (registry $3.54 \pm 1.0$ vs randomized $3.21 \pm 0.9$; $P<.001$ ), corresponding to a significantly higher rate of complete revascularization (registry $75.6 \%$ vs randomized $63.2 \% ; P<.001)$. Of note, surgeons' assessment of vessel diameter and vessel quality at the anastomotic site and distal to the anastomosis were significantly unfavorable in registry patients (data not shown).

\section{Early Results}

Early mortality and MACCE rates were low. Overall hospital mortality was $1.07 \%$ (registry $0.6 \%$ vs randomized $1.4 \% ; P=.144)$ and corresponded to a 30 -day postprocedure mortality of $0.94 \%$ (registry $0.6 \%$ vs randomized $1.2 \% ; P=.252$ ). Hospital and 30-day postprocedure MACCEs were $4.6 \%$ (registry $3.4 \%$ vs randomized $5.4 \%$; $P=.067$ ) and $4.4 \%$ (registry $3.4 \%$ vs randomized $5.2 \%$; $P=.100)$, respectively.

Discharge medications of the entire CABG population were as follows: aspirin $88.5 \%$, B-blocker $78.9 \%$, nitrates $14.0 \%$, angiotensin-converting enzyme inhibitors $44.8 \%$, thienopyridine $18.4 \%$, statins $71.9 \%$, and amiodarone $12.5 \%$; the regimen was not different between registry and randomized patients. Aside from $\beta$-blocker medication and significantly more amiodarone medication $(P<.001)$, patients who underwent $\mathrm{CABG}$ received significantly less pharmacologic treatment $(P<.001$ each $)$ at discharge than did randomized PCI patients. ${ }^{14}$ 
TABLE 1. Baseline characteristics of the CABG study population

\begin{tabular}{|c|c|c|c|c|}
\hline Characteristics & Registry & $(\mathbf{n}=\mathbf{8 9 7})$ & $(n=1541)$ & $\begin{array}{c}P \\
\text { value } \\
\end{array}$ \\
\hline \multicolumn{5}{|l|}{ Extracardiac variables } \\
\hline Age, y & $65.7 \pm 9.4$ & $65.0 \pm 9.8$ & $65.3 \pm 9.6$ & .140 \\
\hline Female, \% & 19.3 & 21.1 & 20.3 & .382 \\
\hline Diabetes, $\%$ & 29.7 & 28.5 & 29.0 & .633 \\
\hline COPD, $\%$ & 7.9 & 9.3 & 8.7 & .359 \\
\hline Arterial hypertension, $\%$ & 73.5 & 77.0 & 75.5 & .114 \\
\hline Hyperlipidemia, \% & 76.4 & 77.2 & 76.9 & .739 \\
\hline $\begin{array}{l}\text { Peripheral vascular } \\
\text { disease, } \%\end{array}$ & 13.8 & 10.6 & 11.9 & .054 \\
\hline $\begin{array}{r}\text { Carotid artery } \\
\text { disease, } \%\end{array}$ & 12.3 & 8.4 & 10.0 & .012 \\
\hline Previous CVA, \% & 5.5 & 4.8 & 5.1 & .571 \\
\hline Smoking history, \% & 64.3 & 69.1 & 67.1 & .050 \\
\hline Body mass index & $28.0 \pm 4.6$ & $27.9 \pm 4.5$ & $27.9 \pm 4.6$ & .746 \\
\hline $\begin{array}{l}\text { Creatinine } \\
\qquad>200 \mu \mathrm{mol} / \mathrm{L}, \%\end{array}$ & 2.0 & 1.8 & 1.9 & .738 \\
\hline Abdominal obesity, $\% *$ & 46.4 & 45.8 & 46.1 & .841 \\
\hline \multicolumn{5}{|l|}{ Cardiac characteristics } \\
\hline Stable angina, $\%$ & 62.9 & 57.2 & 59.6 & .025 \\
\hline Unstable angina, $\%$ & 21.6 & 28.0 & 25.3 & .004 \\
\hline Silent ischemia, $\%$ & 9.0 & 8.2 & 8.6 & .601 \\
\hline No angina, $\%$ & 6.5 & 6.6 & 6.6 & .965 \\
\hline $\begin{array}{l}\text { Emergency } \\
\quad \text { indication, \% }\end{array}$ & 3.6 & 3.9 & 3.8 & .738 \\
\hline Urgent indication, $\%$ & 9.0 & 3.8 & 6.0 & $<.001$ \\
\hline Preoperative IABP, $\%$ & 2.5 & 1.0 & 1.6 & .023 \\
\hline Previous MI, \% & 33.5 & 33.8 & 33.7 & .911 \\
\hline Chronic heart failure, $\%$ & 5.5 & 5.3 & 5.4 & .873 \\
\hline $\begin{array}{l}\text { Pulmonary } \\
\text { hypertension, \% }\end{array}$ & 1.1 & 1.3 & 1.2 & 660 \\
\hline $\begin{array}{l}\text { Ejection fraction } \\
\quad<30 \%, \%\end{array}$ & 4.5 & 2.5 & 3.3 & .030 \\
\hline $\begin{array}{l}\text { Ejection fraction } \\
30 \%-50 \%, \%\end{array}$ & 23.3 & 17.1 & 19.7 & .003 \\
\hline \multicolumn{5}{|l|}{ Coronary complexity } \\
\hline Left dominance, $\%$ & 5.7 & 8.7 & 7.4 & .031 \\
\hline $\begin{array}{l}\text { Diffuse/small } \\
\quad \text { vessel disease, } \%\end{array}$ & 13.6 & 10.7 & 11.9 & .080 \\
\hline LM disease, any, $\%$ & 40.3 & 33.8 & 36.5 & .010 \\
\hline LM disease only, $\%$ & 1.6 & 3.1 & 2.5 & .055 \\
\hline $\mathrm{LM}+1-\mathrm{VD}, \%$ & 2.7 & 5.1 & 4.1 & .017 \\
\hline $\mathrm{LM}+2-\mathrm{VD}, \%$ & 8.4 & 12.0 & 10.5 & .023 \\
\hline $\mathrm{LM}+3-\mathrm{VD}, \%$ & 27.6 & 13.5 & 19.3 & $<.001$ \\
\hline 3-VD only, $\%$ & 59.7 & 66.2 & 63.5 & .010 \\
\hline $\begin{array}{l}\text { Chronic total occlusion, } \\
\text { any, } \% \text { of patients }\end{array}$ & 56.4 & 22.2 & 36.4 & $<.001$ \\
\hline $\begin{array}{l}\text { Chronic total occlusion, } \\
\% \text { of lesions }\end{array}$ & 15.8 & 5.6 & 10.0 & $<.001$ \\
\hline $\begin{array}{r}\text { Bifurcation, any, } \\
\% \text { of patients }\end{array}$ & 80.8 & 73.1 & 76.3 & $<.001$ \\
\hline $\begin{array}{l}\text { Bifurcation, } \% \\
\text { of lesions }\end{array}$ & 30.2 & 27.6 & 28.7 & .020 \\
\hline
\end{tabular}

(Continued)
TABLE 1. Continued

\begin{tabular}{|c|c|c|c|c|}
\hline \multirow[b]{2}{*}{ Characteristics } & \multirow{2}{*}{$\begin{array}{l}\text { Registry } \\
(n=644)\end{array}$} & \multirow{2}{*}{$\begin{array}{c}\text { RCT } \\
(n=897)\end{array}$} & \multirow{2}{*}{$\begin{array}{l}\text { Overall } \\
(n=1541)\end{array}$} & \multirow[b]{2}{*}{$\begin{array}{c}P \\
\text { value } \\
\end{array}$} \\
\hline & & & & \\
\hline $\begin{array}{l}\text { Trifurcation, any, } \\
\% \text { of patients }\end{array}$ & 16.8 & 10.6 & 13.1 & $<.001$ \\
\hline Aorto-ostial lesion & 4.2 & 3.6 & 3.9 & .270 \\
\hline $\begin{array}{l}\text { Trifucation, } \\
\% \text { of lesions }\end{array}$ & 3.6 & 2.4 & 2.9 & .004 \\
\hline $\begin{array}{l}\text { Lesion length } \\
\quad>20 \mathrm{~mm}, \\
\% \text { of lesions }\end{array}$ & 31.5 & 20.3 & 25.1 & $<.001$ \\
\hline $\begin{array}{l}\text { Heavy calcification, } \\
\% \text { of lesions }\end{array}$ & 32.7 & 23.3 & 27.3 & $<.001$ \\
\hline $\begin{array}{l}\text { Severe lesion } \\
\text { tortuosity, } \\
\% \text { of lesions }\end{array}$ & 32.5 & 30.8 & 31.6 & .137 \\
\hline $\begin{array}{l}\text { Thrombus formation, } \\
\% \text { of lesions }\end{array}$ & 1.0 & 1.0 & 1.0 & .854 \\
\hline $\begin{array}{c}\text { Number of coronary } \\
\text { lesions/patient, } n\end{array}$ & $4.6 \pm 1.7$ & $4.4 \pm 1.8$ & $4.5 \pm 1.8$ & .005 \\
\hline$>5$ lesions/patient, $\%$ & 27.5 & 25.7 & 26.5 & .433 \\
\hline SYNTAX score & $37.8 \pm 13.3$ & $29.1 \pm 11.4$ & $32.7 \pm 12.9$ & $<.001$ \\
\hline Total Parsonnet score & $8.96 \pm 7.06$ & $8.43 \pm 6.84$ & $8.65 \pm 6.94$ & .145 \\
\hline Additive EuroSCORE & $3.89 \pm 2.68$ & $3.79 \pm 2.69$ & $3.83 \pm 2.69$ & .466 \\
\hline Logistic EuroSCORE, $\%$ & $4.04 \pm 4.44$ & $3.87 \pm 4.40$ & $3.94 \pm 4.42$ & .463 \\
\hline $\begin{array}{l}R C T \text {, Randomized controlled } \\
C V A \text {, cerebrovascular acciden } \\
\text { infarction; } L M \text {, left main; } V D \text {, } \\
\text { exhibit by core laboratory eva } \\
\text { domized patients. *Abdomina } \\
\text { than } 40 \text { inches in men and gre }\end{array}$ & $\begin{array}{l}\text { l; } C O P D, \mathrm{ch} \\
A B P \text {, intra-aor } \\
\text { ssel disease } \mathrm{C} \\
\text { tion } P \text { value } \mathrm{f} \\
\text { besity was de }\end{array}$ & $\begin{array}{l}\text { ronic obstructiv } \\
\text { rtic balloon pun } \\
\text { Coronary charac } \\
\text { for comparison } \\
\text { efined at waist }\end{array}$ & $\begin{array}{l}\text { ve pulmonary } \\
\text { mping; } M I \text {, my } \\
\text { cteristics were } \\
\text { of registry ver } \\
\text { circumference }\end{array}$ & $\begin{array}{l}\text { disease; } \\
\text { ocardial } \\
\text { given as } \\
\text { sus ran- }\end{array}$ \\
\hline
\end{tabular}

\section{Two-Year Outcome and Relation to SYNTAX Score and Presence of LM Disease}

One- and 2-year MACCE rates of the entire CABG population were $10.9 \%$ and $15.1 \%$, respectively. The incidence of MACCE and all its components was higher during the first than during the second year (Figure 1). Comparing registry and randomized $\mathrm{CABG}$ patients showed a significantly lower MACCE rate at 1 year (registry $8.8 \%$ vs randomized $12.4 \% ; P=.031$ ) and 2 years of follow-up (registry $13.0 \%$ vs randomized $16.7 \% ; P=.046$ ) for registry patients. This was exclusively based on differences in the need for repeat revascularization, which at 1 year (registry $3.0 \%$ vs randomized $5.9 \% ; P=.009$ ) and 2 years (registry $4.7 \%$ vs randomized $8.6 \% ; P=.004$ ) was nearly twice as high for randomized as for registry patients (Table 3 ).

With regard to the SYNTAX score, the overall MACCE rates at 2 years in patients with low $(0-22 ; 15.6 \%)$, intermediate $(23-32 ; 14.3 \%)$, and high $(\geq 33 ; 15.4 \%)$ SYNTAX scores and all MACCE components were similar (Figure 2, Table 3). By comparing registry and randomized patients, we found a significantly reduced 2-year MACCE rate for registry patients with low (registry $7.5 \%$ vs randomized $17.8 \% ; P=.048)$ and intermediate 
TABLE 2. Treatment of CABG patients (registry versus randomized patients)

\begin{tabular}{|c|c|c|c|c|}
\hline & Registry & RCT & Overall & \\
\hline & $(n=644)$ & $(\mathbf{n}=\mathbf{8 9 7})$ & $(n=1541)$ & $P$ value \\
\hline Arterial graft to LAD & 94.7 & 95.6 & 95.2 & .457 \\
\hline BITA use, $\%$ & 16.1 & 27.6 & 22.7 & $<.001$ \\
\hline Radial artery use, $\%$ & 11.2 & 14.1 & 12.8 & .100 \\
\hline Gastroepiploic artery use, $\%$ & 0.0 & 0.0 & 0.0 & \\
\hline Complete arterial, $\%$ & 11.2 & 18.9 & 15.6 & $<.001$ \\
\hline At least one arterial graft, $\%$ & 96.7 & 97.3 & 97.1 & .520 \\
\hline More than one arterial graft, $\%$ & 26.2 & 35.3 & 31.5 & $<.001$ \\
\hline Venous grafts only, $\%$ & 3.3 & 2.6 & 2.9 & .432 \\
\hline Grafts per patient, $\mathrm{n}$ & $3.0 \pm 0.9$ & $2.8 \pm 0.7$ & $2.9 \pm 0.8$ & $<.001$ \\
\hline Arterial & $1.3 \pm 0.7$ & $1.4 \pm 0.7$ & $1.4 \pm 0.7$ & .001 \\
\hline Venous & $1.7 \pm 1.0$ & $1.4 \pm 0.8$ & $1.5 \pm 1.0$ & $<.001$ \\
\hline Distal anastomoses/patient, $\mathrm{n}$ & $3.5 \pm 1.0$ & $3.2 \pm 0.9$ & $3.4 \pm 0.9$ & $<.001$ \\
\hline Graft to LAD territory, any, \% & 99.1 & 99.8 & 99.5 & .082 \\
\hline Graft to $\mathrm{Cx}$ territory, any, \% & 93.0 & 92.5 & 92.7 & .709 \\
\hline Graft to RCA territory, any $\%$ & 75.8 & 71.8 & 73.4 & .096 \\
\hline Complete revascularization, $\%$ & 75.6 & 63.2 & 68.1 & $<.001$ \\
\hline Endarterectomy, any, \% & 1.8 & 1.2 & 1.5 & .064 \\
\hline Dist. anastomosis from Y-graft, $\%$ & 3.5 & 7.7 & 5.8 & $<.001$ \\
\hline Dist. anastomosis from jump-graft, $\%$ & 30.4 & 25.0 & 27.5 & .001 \\
\hline OPCAB, $\%$ & 18.6 & 15.0 & 16.6 & .062 \\
\hline Cardioplegia use, $\%$ & 78.4 & 82.7 & 80.9 & .041 \\
\hline Blood cardioplegia, $\%$ & 41.9 & 50.1 & 46.6 & .002 \\
\hline Crystalloid cardioplegia, \% & 36.5 & 32.6 & 34.3 & .116 \\
\hline Procedure time, $\mathrm{h}$ & $3.6 \pm 1.0$ & $3.4 \pm 1.1$ & $3.5 \pm 1.1$ & .018 \\
\hline Bypass time, min & $93.8 \pm 35.0$ & $86.5 \pm 35.2$ & $89.5 \pm 35.3$ & $<.001$ \\
\hline Crossclamp time, $\min$ & $60.0 \pm 26.8$ & $55.0 \pm 24.9$ & $57.0 \pm 25.8$ & .001 \\
\hline Aprotinin given, $\%$ & 32.9 & 36.7 & 35.1 & .130 \\
\hline Intra-aortic balloon pump, $\%$ & 4.8 & 2.9 & 3.7 & .057 \\
\hline$\Delta \mathrm{t}$ consent/revascularization, $\mathrm{d}$ & 8.1 & 17.4 & 13.6 & $<.001$ \\
\hline Postprocedural hospital stay, d & $8.2 \pm 5.7$ & $9.5 \pm 8.0$ & $9.0 \pm 7.2$ & .001 \\
\hline
\end{tabular}

$C A B G$, Coronary artery bypass grafting; $R C T$, randomized controlled trial; $L A D$, left anterior descending coronary artery; $B I T A$, bilateral internal thoracic artery; $C x$, circumflex; $R C A$, right coronary artery; $O P C A B$, off-pump coronary artery bypass; $\Delta t$, time interval randomization/revascularization in days.

scores (registry $9.5 \%$ vs randomized $17.0 \% ; P=.042$ ), but not with high SYNTAX scores (registry $15.2 \%$ vs randomized $15.7 \% ; P=.832$ ). In the intermediate SYNTAX score population, this difference reached significance regarding death $(P=.037)$ and repeat revascularization $(P=.037)$.

The 2-year MACCE rate in LM patients was significantly higher than in patients with isolated 3-VD (LM 17.0\% vs 3 -VD $13.2 \% ; P=.042)$. There was also a trend for higher mortality (LM $5.9 \%$ vs 3 -VD $4.1 \% ; P=.082$ ) and symptomatic graft occlusion (LM $3.9 \%$ vs 3 -VD $2.1 \%$; $P=.063) 2$ years postoperatively.

A significant difference was demonstrated between results in patients treated in the US (261 patients) and outside the US (OUS, 1280 patients). Rate of repeat revascularization after 2 years was significantly higher in the US compared with the OUS cohort (US $11.6 \%$ vs OUS $6.0 \% ; P=.002)$. Overall MACCE rate was significantly higher (US $19.4 \%$ vs OUS $14.3 \% ; P=.04$ ), and all other components were slightly lower (death: US $4.5 \%$, OUS
$5.1 \% ; P=.738$; MI: US $2.1 \%$, OUS $3.5 \% ; P=0.249$; CVA: US $2.8 \%$, OUS $3.7 \% ; P=.425$ ) in the US.

\section{Univariate and Multivariate Logistic Regression Model}

Twenty-four of 110 analyzed variables had a significant univariate or multivariate association with 2-year MACCE rate (Table 4). Widely known extracardiac and cardiac risk factors (ie, arterial hypertension, EuroSCORE, peripheral vascular disease, advanced age, chronic obstructive pulmonary disease, low ejection fraction, intra-aortic balloon pumping, and emergency indication) adversely affected long-term CABG outcomes, whereas medically treated diabetes was not a significant risk factor $(P=.201)$. Concerning coronary complexity, neither the raw SYNTAX score $(P=.786)$ nor most of its elements (ie, birfurcation/trifurcation; $P=.141$; lesion length $>20$ $\mathrm{mm} ; P=.217$; diffuse coronary artery disease; $P=.224$; number of lesions; $P=.375$; heavy calcification, $P=.705$; aorto-ostial lesion; $P=.825$; left dominance; 


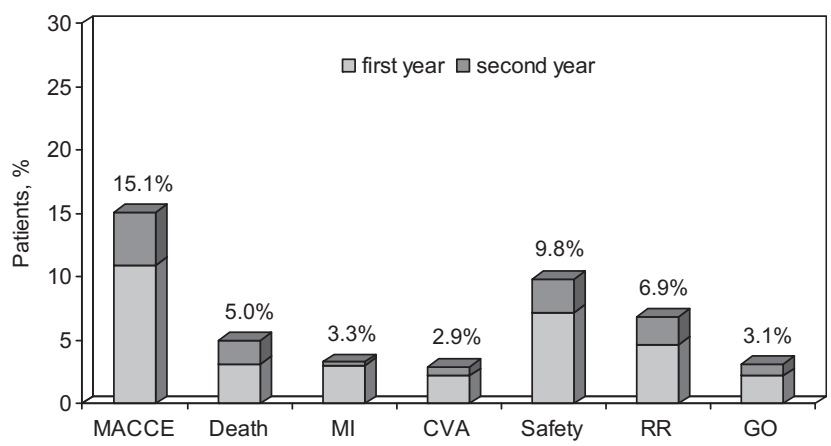

FIGURE 1. Overall 2-year MACCE rate and MACCE components of the entire SYNTAX CABG study population $(n=1468)$, divided by incidence during the first and second year after allocation. MACCE, Major adverse cardiac and cerebrovascular event; $M I$, myocardial infarction; $C V A$, cerebrovascular event; $R R$, repeat revascularization; $G O$, symptomatic graft occlusion. Safety end point was defined as combined end point of Death, $\mathrm{MI}$ and CVA.

$P=.837$; any chronic total occlusion; $P=.880$; severe tortuosity; $P=.916$ ) influenced 2-year MACCE rate. The presence of coronary thrombus formation and LM disease (particularly in combination with 2-VD) were significantly associated with MACCE at 2 years (Table 4).

Concerning the bypass surgical strategy, neither offpump coronary bypass $(P=.829)$ nor blood cardioplegia use $(P=.281)$ significantly influenced 2 -year results, but procedural time was associated with worse outcomes. Of note, the choice of additional arterial grafts (right ITA; $P=.840$; radial artery; $P=.320$ ) and complex graft constructions (jump-graft; $P=.500$; Y-graft; $P=.341$ ) also did not significantly affect outcome, but there was a trend toward improved results with an increasing number of distal arterial anastomoses $(P=.311)$.

In contrast, complete surgical revascularization was significantly protective against 2-year MACCE (complete $13.0 \%$ vs incomplete $18.3 \% ; P=.007$ ) (Figure 3 ). In addition, the total number of distal anastomoses $(P=.01)$, conduits $(P=.022)$, and treated vessels $(P=.022)$ had a beneficial effect on outcomes (Table 4 ).

\section{DISCUSSION}

SYNTAX was designed to compare modern surgical and percutaneous techniques as a primary treatment strategy for patients with de novo 3-VD or LM disease. Inasmuch as CABG proved to be superior with regard to the primary end point of 12-month MACCE,${ }^{14}$ the aim of the current study was to describe the contemporary CABG standard of care and to identify clinical and coronary risk factors for adverse surgical outcome with a special focus on the impact of coronary complexity. Of note, this population is the largest surgical cohort analyzed in a single controlled multicenter clinical trial to date.

TABLE 3. Incidence of major adverse cardiac and cerebrovascular events at 1- and 2-year follow-up divided by raw SYNTAX score and CABG study arm

\begin{tabular}{|c|c|c|c|c|c|c|c|c|}
\hline \multirow[b]{2}{*}{ SYNTAX score } & \multicolumn{4}{|c|}{ One year } & \multicolumn{4}{|c|}{ Two years } \\
\hline & $\begin{array}{c}\text { Overall } \\
(n=1522)\end{array}$ & $\begin{array}{c}0-22 \\
(n=343)\end{array}$ & $\begin{array}{c}23-32 \\
(n=461)\end{array}$ & $\begin{array}{c}\geq 33 \\
(\mathbf{n}=\mathbf{7 1 8})\end{array}$ & Overall & 0-22 & 23-32 & $\geq \mathbf{3 3}$ \\
\hline MACCE, $\%$ & 10.9 & 12.8 & 9.7 & 10.8 & 15.1 & 15.6 & 14.3 & 15.4 \\
\hline Randomized & 12.4 & 14.6 & 12.0 & 10.9 & 16.7 & 17.8 & 17.0 & 15.7 \\
\hline Registry & $8.8^{*}$ & 6.0 & $5.6^{*}$ & 10.6 & $13.0 *$ & $7.5^{*}$ & $9.5^{*}$ & 15.2 \\
\hline Death, $\%$ & 3.1 & 3.1 & 3.2 & 3.2 & 5.0 & 4.6 & 4.8 & 5.2 \\
\hline Randomized & 3.5 & 3.8 & 4.2 & 2.6 & 5.0 & 5.4 & 6.5 & 3.3 \\
\hline Registry & 2.5 & 0 & 1.3 & 3.5 & 4.9 & 1.5 & $1.9^{*}$ & 6.6 \\
\hline MI, \% & 3.0 & 2.8 & 2.5 & 3.4 & 3.3 & 2.8 & 2.8 & 3.7 \\
\hline Randomized & 3.2 & 3.5 & 2.5 & 4.0 & 3.5 & 3.5 & 2.9 & 4.0 \\
\hline Registry & 2.5 & 0 & 2.5 & 3.0 & 3.0 & 0.0 & 2.5 & 3.5 \\
\hline CVA, $\%$ & 2.2 & 2.1 & 2.5 & 2.2 & 2.9 & 3.1 & 3.0 & 2.7 \\
\hline Randomized & 2.2 & 1.9 & 2.5 & 2.3 & 2.9 & 2.7 & 2.9 & 3.0 \\
\hline Registry & 2.2 & 3.0 & 2.5 & 2.0 & 3.0 & 4.5 & 3.2 & 2.5 \\
\hline Safety end point, $\%$ & 7.2 & 6.7 & 7.0 & 7.7 & 9.8 & 8.9 & 9.4 & 10.4 \\
\hline Randomized & 7.7 & 7.7 & 7.7 & 7.6 & 9.9 & 10.0 & 10.9 & 9.0 \\
\hline Registry & 6.6 & 3.0 & 5.6 & 7.8 & 9.7 & 4.5 & 7.0 & 11.4 \\
\hline Repeat revascularization, $\%$ & 4.7 & 6.4 & 4.1 & 4.2 & 6.9 & 7.4 & 7.1 & 6.5 \\
\hline Randomized & 5.9 & 7.3 & 5.6 & 5.0 & 8.6 & 8.5 & 9.1 & 8.4 \\
\hline Registry & $3.0^{*}$ & 3.0 & $1.3^{*}$ & 3.5 & $4.7^{*}$ & 3.0 & $3.8^{*}$ & 5.1 \\
\hline Sympt. graft occlusion, $\%$ & 2.2 & 3.6 & 2.6 & 2.4 & 3.1 & 2.7 & 2.7 & 3.3 \\
\hline Randomized & 2.1 & 4.2 & 4.2 & 2.1 & 2.6 & 3.0 & 2.8 & 2.2 \\
\hline Registry & 2.4 & 1.5 & 0 & 2.7 & 3.7 & 1.5 & 2.6 & 4.1 \\
\hline
\end{tabular}

$C A B G$, Coronary artery bypass graft; $M A C C E$, major adverse cardiac and cerebrovascular event; $M I$, myocardial infarction; $C V A$, cerebrovascular accident. $* P<.05$ for randomized versus registry $C A B G$ patients. 


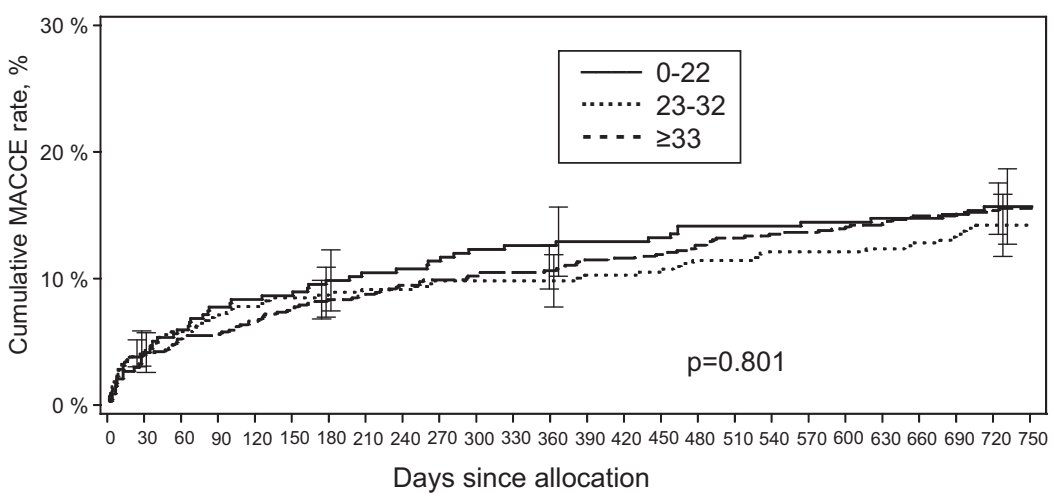

FIGURE 2. MACCE in CABG patients increased not with high raw SYNTAX scores. Kaplan-Meier curves for 2-year MACCE in CABG divided by raw SYNTAX score. Patients with low $(0-22 ; 15.6 \%)$, intermediate $(23-32 ; 14.3 \%)$, and high $(\geq 33 ; 15.4 \%)$ SYNTAX scores have similar 2-year MACCE rates. Numbers in the figure are cumulative event rates \pm 1.5 standard error. MACCE, Major adverse cardiac and cerebrovascular event.

Surgical data from 85 sites in Europe and the US revealed a conventional surgical strategy with cardioplegic arrest and a single arterial bypass graft in the vast majority of patients. This is noteworthy because surgical study recommendations included a liberal use of bilateral ITA, particularly in patients less than 70 years of age. These recommendations were based on the knowledge of superior bilateral ITA long-term results compared with venous bypass graft- ing. ${ }^{19}$ However, a more conservative treatment philosophy in the majority of centers had to be recognized, which somehow still reflects the real world of CABG surgery, despite the fact that all participating sites were encouraged for liberal use of double ITA grafting. Interestingly, the analysis of the entire SYNTAX CABG population revealed no evidence of a significant advantage of arterial grafting over venous bypass surgery in the presence of an ITA-to-LAD

TABLE 4. Univariate and multivariate logistic regression for MACCE at 2 years after allocation including all SYNTAX CABG patients

\begin{tabular}{|c|c|c|c|c|c|c|}
\hline \multirow[b]{2}{*}{ Characteristics } & \multicolumn{3}{|c|}{ Univariate } & \multicolumn{3}{|c|}{ Multivariate } \\
\hline & OR & CI & $\boldsymbol{P}$ & OR & CI & $P$ \\
\hline Arterial hypertension & 2.15 & $1.45-3.18$ & $<.001$ & 1.86 & $1.07-3.23$ & .027 \\
\hline EuroSCORE & 1.10 & $1.05-1.16$ & $<.001$ & & & \\
\hline Perioperative IABP & 2.97 & $1.65-5.32$ & $<.001$ & 2.28 & $1.12-4.64$ & .023 \\
\hline Peripheral vascular disease & 1.88 & $1.28-2.76$ & .001 & 1.93 & $1.18-3.16$ & .000 \\
\hline Complete revascularization & 0.66 & $0.49-0.88$ & .005 & 0.66 & $0.51-0.86$ & .002 \\
\hline Emergency indication & 2.34 & $1.29-4.26$ & .005 & 2.35 & $1.13-4.88$ & .022 \\
\hline CCS class & 1.41 & $1.10-1.80$ & .006 & 1.63 & $1.05-2.53$ & .031 \\
\hline COPD & 1.81 & $1.17-2.80$ & .008 & & & \\
\hline Age & 1.02 & $1.01-1.03$ & .009 & & & \\
\hline No. of distal anastomoses & 0.81 & $0.69-0.95$ & .010 & & & \\
\hline IV inotropes periprocedural & 1.42 & $1.06-1.91$ & .018 & & & \\
\hline No. of total conduits & 0.81 & $0.67-0.97$ & .022 & & & \\
\hline No. of vessels treated & 0.73 & $0.55-0.96$ & .022 & & & \\
\hline Systolic blood pressure & 1.01 & $1.00-1.01$ & .027 & & & \\
\hline Ejection fraction $<30 \%$ & 1.41 & $1.02-1.94$ & .035 & & & \\
\hline Previous TIA & 1.78 & $1.03-3.07$ & .040 & & & \\
\hline Procedure time & 1.14 & $1.01-1.30$ & .041 & 1.32 & $1.11-1.57$ & .002 \\
\hline Allocation in the United States & 1.45 & $1.02-2.07$ & .042 & 1.84 & $1.14-2.96$ & .012 \\
\hline Randomization & 1.35 & $1.00-1.81$ & .046 & & & \\
\hline LM disease, any & 1.33 & $1.00-1.77$ & .049 & & & \\
\hline Diastolic blood pressure & 1.01 & $1.00-1.02$ & .051 & 1.02 & $1.00-1.04$ & .018 \\
\hline Intracoronary thrombus formation & 1.84 & $0.99-3.41$ & .054 & 2.81 & $1.26-6.26$ & .012 \\
\hline Preoperative ACE inhibitor & 1.31 & $0.98-1.75$ & .064 & & & \\
\hline $\mathrm{LM}+2-\mathrm{VD}$ & 1.46 & $0.97-2.21$ & .070 & 1.62 & $0.95-2.78$ & .079 \\
\hline
\end{tabular}

Depicted are all variables with $P<.1$ in univariate analysis in order of probability. $M A C C E$, major adverse cardiac and cerebrovascular event; $C A B G$. coronary artery bypass grafting; $O R$, odds ratio; $C I$, $95 \%$ confidence interval; IABP, intra-aortic balloon pump; $C C S$, Canadian Cardiovascular Society; COPD, chronic obstructive pulmonary disease; $I V$, intravenous; $T I A$, transient ischemic attack; $L M$, left main; $A C E$, angiotensin-converting enzyme; $V D$, vessel disease. 
anastomosis. Thus, further long-term results are needed to verify the expected long-term benefit of bilateral ITA and arterial bypass surgery in the context of a controlled clinical multicenter trial.

An important conclusion from the current study is that contemporary CABG results are excellent, with an overall early mortality rate of about $1 \%$. This very low mortality rate is even more remarkable given that only a few study exclusion criteria were defined. However, there were a significant number of cerebrovascular events in the perioperative and early postoperative period, which may be seen as the "Achilles heel" of modern bypass surgery. All efforts need to be made to significantly reduce this adverse event in the future, inasmuch as it might serve as one major reason to decline CABG as the primary treatment option. We also need to recognize the significantly decreased administration of known beneficial discharge medications (ie, angiotensinconverting enzyme inhibitors, statins, aspirin) in surgical versus interventional patients. Thus, our intent had to be that cardiac medication be optimized at discharge and continued during follow-up in all surgical patients.

The SYNTAX score was initially designed as a comprehensive angiographic scoring system to anticipate PCI complexity related to coronary disease and to allow quantification of coronary artery disease beyond LM and major 3-VD. ${ }^{15}$ The SYNTAX score was first retrospectively validated on 306 patients who underwent PCI for 3-VD disease in the ARTS II study population and was found to have great discrimination ability for MACCE 1 year after the procedure. ${ }^{16}$ As well, the raw SYNTAX score in randomized patients receiving PCI during the SYNTAX trial was highly predictive of adverse outcomes, indicating that high SYNTAX scores can serve as a determent for the percutaneous approach. ${ }^{14,20}$

In contrast, the predictive value of the SYNTAX score and its components for CABG patients is not clear. Although the SYNTAX score did not give direction for 1-year MACCE in the randomized CABG cohort of the SYNTAX study, more recently it was found to be indicative for MACCE in a surgical population of LM patients. ${ }^{14,18}$

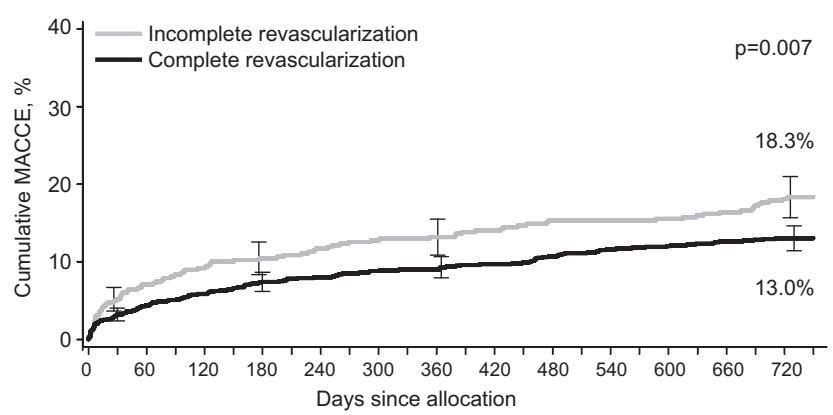

FIGURE 3. Two-year freedom from MACCE of SYNTAX coronary artery bypass patients comparing complete and incomplete revascularization. MACCE, Major adverse cardiac and cerebrovascular event.
Additionally, Capodonno and coworkers ${ }^{17}$ describe a difference in 2-year mortality rates in LM surgical patients of $6.2 \%$ for SYNTAX scores of 34 or less and $8.5 \%$ for scores above 34 . These results, however, need to be assessed against the background of a mortality difference in PCI treatment of $8.1 \%$ versus $32.7 \%{ }^{17}$ We herein confirmed the results of the randomized SYNTAX study population by finding that 2-year postoperative outcomes were not influenced by differences in the raw SYNTAX score.

Even though the SYNTAX score is not as important for surgery as for PCI, the existence of a threshold to define which patients should be referred for surgery remains unclear. The separation into tertiles of less than 22, 22 to 32, and 32 or more, used in the SYNTAX trial, was not based on clinical considerations, but on the statistical requirement for each tertile to include an equal number of patients. This was also the reason that the thresholds varied (SYNTAX score level of 19/25, 18/26, and 34) in other studies. ${ }^{16-18}$ Another limitation of the SYNTAX score is its moderate interobserver and intraobserver reproducibility of the score. $^{21,22}$ Additionally, it remains unknown whether the raw SYNTAX score or individual score components are more indicative of adverse outcomes. At least for the surgical SYNTAX CABG population, we found the presence of intracoronary thrombus formation and LM disease rather than the raw SYNTAX score as significant predictors for 2-year MACCE, whereas important PCI variables like lesion length, chronic total vessel occlusion, bifurcation, vessel tortuosity, and calcifications were not associated with adverse outcomes. However, additional validation of the SYNTAX score and its components in other patient populations, clinical scenarios, and for other end points are required.

We found a significantly worse outcome of randomized compared with registry patients in groups of low and intermediate SYNTAX scores that was predominantly based on differences in the rate of repeat revascularization. This finding was unexpected, because randomized patients were considered to be healthier and to have less complex coronary disease. Indeed, SYNTAX scores were higher in registry patients, but extracardiac and clinical cardiac variables were evenly balanced between both study populations. Because the SYNTAX score itself did not significantly influence 2-year results, other surgical variables had to be analyzed to explain these notable outcome difference. During univariate analyses, we found that neither off-pump coronary bypass surgery nor the choice of arterial grafts in addition to ITA-to-LAD anastomosis influenced outcome. However, there was a significant difference in completeness of revascularization with an $11 \%$ absolute higher rate of complete revascularization in registry compared with randomized patients (registry $74.7 \%$ vs randomized $63.2 \%$; $P<.001$ ). As a surrogate for completeness of revascularization, we also found a significantly higher number of grafts 
and distal anastomoses per patient in the registry, which were independently protective against 2-year MACCE rates.

Different definitions of completeness of revascularization exist in the literature. ${ }^{23}$ In the SYNTAX study, the functional classification requiring $\mathrm{CABG}$ to all major diseased and graftable coronary segments presenting significant flow-compromising lesions was used. This definition has been advocated in other randomized CABG versus PCI studies. ${ }^{24}$ The use of this definition, among others, might be one reason for the comparably low rate of complete revascularization found in the SYNTAX trial compared with other studies in which the traditional definition was used. $^{14,23}$

The impact of completeness of revascularization in contemporary bypass surgery is still a matter of debate. It was recently shown that in presence of a left ITA-to-LAD anastomosis, a reasonably incomplete surgical revascularization of the circumflex or right coronary artery territory did not adversely affect early or long-term survival in patients with multivessel coronary artery disease. ${ }^{23,24}$ However, the data from the present analysis clearly suggest the high impact of incomplete surgical revascularization on the requirement for repeat revascularization during the 2-year follow-up, even if survival might not yet be significantly affected.

In contrast to surgery, there is some evidence that patients undergoing PCI who receive incomplete revascularization experience more death with bare metal stents and possibly also with drug-eluting stents. ${ }^{24,25}$ Thus, it must be stressed that the rate of surgical incomplete revascularization is markedly different from that in PCI of multivessel disease, and this has to be considered during the primary treatment decision. ${ }^{25}$

We also found a surprisingly higher 2-year MACCE rate in patients treated in the US compared with OUS (US $19.4 \%$ vs OUS $14.3 \% ; P=.04)$. This was exclusively explained by a notably higher rate of repeat revascularization, whereas there was a trend of lower incidence of safety end points in the US CABG population. Reasons for these findings are speculative, and differences might be explained by a significantly lower number of included patients per center, a more aggressive use of postoperative coronary angiography, and a higher percentage of LM patients.

\section{LIMITATIONS}

Although this study provides important information about contemporary coronary bypass surgery, there are limitations. Two years of follow-up does not adequately reflect the true long-term effect of arterial grafts over veins regarding patency and the need for reintervention.

Most analyses were applied to the overall 2-year MACCE rate, keeping in mind that most differences were found for the event of repeat revascularization and less for death, CVA, or MI. A higher rate of repeat revascularization does not per se indicate a higher incidence of recurrent angina, but it could also only reflect the presence of a vessel feasible for PCI after initial CABG in a less complex coronary pathologic condition.

The post hoc SYNTAX score subgroup analyses were not prespecified or statistically powered.

\section{CONCLUSIONS}

Surgical patients from the SYNTAX trial form an ideal population to study the results of current surgical CABG practice. Outcome data reveal very low early and followup mortality rates, whereas CVA was relatively high. The raw Syntax score was not predictive of 2-year MACCE, but patients with LM stenosis were at increased risk. Complete surgical revascularization is a major determinant for event-free long-term outcome.

\section{References}

1. Patel MR, Dehmer GJ, Hirshfeld JW, Smith PK, Spertus JA, et al. American College of Cardiology Foundation Appropriateness Criteria Task Force; Society for Cardiovascular Angiography and Interventions; Society of Thoracic Surgeons; American Association for Thoracic Surgery; American Heart Association, and the American Society of Nuclear Cardiology Endorsed by the American Society of Echocardiography; Heart Failure Society of America; Society of Cardiovascular Computed Tomography. ACCF/SCAI/STS/AATS/AHA/ASNC 2009 Appropriateness Criteria for Coronary Revascularization. J Am Coll Cardiol. 2009;53: 530-3.

2. Fox K, Garcia MA, Ardissino D, Buszman P, Camici PG, Crea F, et al. Guidelines on the management of stable angina pectoris: executive summary: The Task Force on the Management of Stable Angina Pectoris of the European Society of Cardiology. Eur Heart J. 2006;27:1341-81.

3. King SB 3rd, Smith SC Jr, Hirshfeld JW Jr, Jacobs AK, Morrison DA, Williams DO, et al. ACC/AHA/SCAI, Adams CD, Anderson JL, Buller CE, Creager MA, Ettinger SM, Halperin JL, Hunt SA, Krumholz HM, Kushner FG, Lytle BW, Nishimura R, Page RL, Riegel B, Tarkington LG, Yancy CW. 2007 Focused Update of the ACC/AHA/SCAI 2005 Guideline Update for Percutaneous Coronary Intervention: A report of the American College of Cardiology/American Heart Association Task Force on Practice Guidelines: 2007 Writing Group to Review New Evidence and Update the ACC/AHA/SCAI 2005 Guideline Update for Percutaneous Coronary Intervention, Writing on Behalf of the 2005 Writing Committee. Circulation. 2008;117:261-95.

4. Eagle KA, Guyton RA, Davidoff R, Edwards FH, Ewy GA, Gardner TJ, et al. American College of Cardiology; American Heart Association. ACC/AHA 2004 guideline update for coronary artery bypass graft surgery: a report of the American College of Cardiology/American Heart Association Task Force on Practice Guidelines (Committee to Update the 1999 Guidelines for Coronary Artery Bypass Graft Surgery). Circulation. 2004;110:e340-437.

5. Hlatky MA, Boothroyd DB, Bravata DM, Boersma E, Booth J, Brooks MM, et al. Coronary artery bypass surgery compared with percutaneous coronary interventions for multivessel disease: a collaborative analysis of individual patient data from ten randomised trials. Lancet. 2009;373:1190-7.

6. Booth J, Clayton T, Pepper J, Nugara F, Flather M, Sigwart U, et al., SoS Investigators. Randomized, controlled trial of coronary artery bypass surgery versus percutaneous coronary intervention in patients with multivessel coronary artery disease: six-year follow-up from the Stent or Surgery Trial (SoS). Circulation. 2008;118:381-8.

7. Daemen J, Boersma E, Flather M, Booth J, Stables R, Rodriguez A, et al. Longterm safety and efficacy of percutaneous coronary intervention with stenting and coronary artery bypass surgery for multivessel coronary artery disease: a metaanalysis with 5-year patient-level data from the ARTS, ERACI-II, MASS-II, and SoS trials. Circulation. 2008;118:1146-54.

8. Hannan EL, Wu C, Walford G, Culliford AT, Gold JP, Smith CR, et al. Drugeluting stents vs. coronary-artery bypass grafting in multivessel coronary disease. N Engl J Med. 2008;358:331-41.

9. Kappetein AP, Dawkins KD, Mohr FW, Morice MC, Mack MJ, Russell ME, et al. Current percutaneous coronary intervention and coronary artery bypass grafting 
practices for three-vessel and left main coronary artery disease. Insights from the SYNTAX run-in phase. Eur J Cardiothorac Surg. 2006;29:486-91.

10. Serruys PW, Onuma Y, Garg S, Vranckx P, De Bruyne B, Morice MC, et al. ARTS II Investigators. 5-year clinical outcomes of the ARTS II (Arterial Revascularization Therapies Study II) of the sirolimus-eluting stent in the treatment of patients with multivessel de novo coronary artery lesions. J Am Coll Cardiol. 2010;55:1093-101.

11. Javaid A, Steinberg DH, Buch AN, Corso PJ, Boyce SW, Pinto Slottow TL, et al. Outcomes of coronary artery bypass grafting versus percutaneous coronary intervention with drug-eluting stents for patients with multivessel coronary artery disease. Circulation. 2007;116(11 Suppl):I200-6.

12. Chieffo A, Morici N, Maisano F, Bonizzoni E, Cosgrave J, Montorfano M, et al. Percutaneous treatment with drug-eluting stent implantation versus bypass surgery for unprotected left main stenosis: a single-center experience. Circulation. 2006;113:2542-7.

13. Ong AT, Serruys PW, Mohr FW, Morice MC, Kappetein AP, Holmes DR Jr, et al. The SYNergy between percutaneous coronary intervention with TAXus and cardiac surgery (SYNTAX) study: design, rationale, and run-in phase. Am Heart J. 2006; 151:1194-204

14. Serruys PW, Morice MC, Kappetein AP, Colombo A, Holmes DR, Mack MJ, et al. SYNTAX Investigators. Percutaneous coronary intervention versus coronary-artery bypass grafting for severe coronary artery disease. $N$ Engl J Med. 2009;360:961-72.

15. Sianos G, Morel MA, Kappetein AP, Morice MC, Colombo C, Dawkins K, et al. The SYNTAX Score: an angiographic tool grading the complexity of coronary artery disease. EuroIntervention. 2005;1:219-27.

16. Valgimigli M, Serruys PW, Tsuchida K, Vaina S, Morel MA, van den Brand MJ, et al. Cyphering the complexity of coronary artery disease using the SYNTAX score to predict clinical outcome in patients with three-vessel lumen obstruction undergoing percutaneous coronary intervention. Am J Cardiol. 2007;99:1072-81.

17. Capodanno D, Capranzano P, Di Salvo ME, Caggegi A, Tomasello D, Cincotta G, et al. Usefulness of SYNTAX score to select patients with left main coronary artery disease to be treated with coronary artery bypass graft. JACC Cardiovasc Interv. 2009;2:731-8.

18. Birim O, van Gameren M, Bogers AJ, Serruys PW, Mohr FW, Kappetein AP. Complexity of coronary vasculature predicts outcome of surgery for left main disease. Ann Thorac Surg. 2009;87:1097-104.

19. Lytle BW, Blackstone EH, Sabik JF, Houghtaling P, Loop FD, Cosgrove DM. The effect of bilateral internal thoracic artery grafting on survival during 20 postoperative years. Ann Thorac Surg. 2004;78:2005-12.

20. Dawkins KD, Morel MA, Serruys PW. Counting the score: The SYNTAX Score and coronary risk. EuroIntervention. 2009;5:33-5.

21. Serruys PW, Onuma Y, Garg S, Sarno G, van den Brand M, Kappetein AP, et al. Assessment of the SYNTAX Score in the SYNTAX study. EuroIntervention. 2009;5:50-6.

22. Garg S, Girasis C, Sarno G, Goedhart D, Morel MA, Garcia-Garcia HM, et al., on behalf of the SYNTAX trial investigators. The SYNTAX score revisited: a reassessment of the SYNTAX Score reproducibility. Catheter Cardiovasc Interv. 2010;75:946-52.

23. Rastan AJ, Walther T, Falk V, Kempfert J, Merk D, Lehmann S, et al. Does reasonable incomplete surgical revascularization affect early or long-term survival in patients with multi-vessel coronary artery disease receiving left internal mammary artery to left anterior descending artery bypass? Circulation. 2009;120: S70-7.

24. van den Brand MJ, Rensing BJ, Morel MA, Foley DP, de Valk V, Breeman A, et al. The effect of completeness of revascularization on event-free survival at one year in the ARTS trial. J Am Coll Cardiol. 2002;39:559-64.

25. Hannan EL, Wu C, Walford G, Holmes DR, Jones RH, Sharma S, et al. Incomplete revascularization in the era of drug-eluting stents: impact on adverse outcomes. JACC Cardiovasc Interv. 2009;2:17-25.

\section{Discussion}

Dr Sheng-Shou Hu (Beijing, China). Congratulations, Dr Rastan, for your excellent presentation.

SYNTAX is a widely known study comparing outcomes of CABG with PCI in patients with 3-VD or LM disease. The purpose of the current study is to describe the current status of CABG based on the SYNTAX CABG cohort and to identify risk factors for adverse outcomes with a special focus on the impact of coronary complexity quantified by SYNTAX score. As far as we know, the SYNTAX CABG cohort is the largest one analyzed in a single controlled multicenter clinical trial right now.

One important conclusion from the current study is the excellent contemporary $\mathrm{CABG}$ results. Outcome data demonstrate very low early and follow-up mortality rates. However, CVAs were relatively common for CABG patients, which may be said to be a big limitation of modern $\mathrm{CABG}$ when compared with PCI to treat 3-VD or LM chronic disease. Therefore, we cardiac surgeons need to spare more effort to reduce CVAs in the future.

The SYNTAX score was initially designed as a comprehensive angiographic scoring system to anticipate PCI complexity related to coronary disease, and it can serve as risk stratification and risk prediction for PCI patients. However, the predictive value of the SYNTAX score for CABG patients is unclear. A recent study from another group in your home country, a paper published in the Annals of Thoracic Surgery last year, found that SYNTAX score was indicative of 1-year outcomes in CABG patients. However, your study found that 2-year post-CABG outcomes were not affected by the SYNTAX score. Therefore, future validation of the SYNTAX score in other patient populations is required.

I have 3 questions. As I just mentioned, a previous study by Dr Ozcan Birim found that SYNTAX score was indicative for 1-year outcomes in CABG patients. How do you explain the difference between your findings and his?

Dr Rastan. We mentioned this paper from the Rotterdam group in the paper discussion. This study was a single-center analysis on patients with LM disease, and data suggested that the SYNTAX score has an impact on the surgical result in about 150 patients. As you could read, this was hardly discussed afterward even by the coauthors. I am, however, sure that the SYNTAX trial data, having 10-fold more patients available, are more convincing and clearly demonstrated that the SYNTAX score itself does not affect the results of CABG.

Dr Hu. Second, we found significantly less completeness of coronary revascularization and worse outcomes of randomized patients compared with registry patients. This finding was quite unexpected and surprising, because registry patients were more likely to have had LM disease and a higher SYNTAX score, whereas randomized patients were more likely to have had a bilateral ITA used and more arterial grafts. Why were randomized patients with lower SYNTAX score associated with less complete coronary revascularization and adverse outcomes? What is your comment on that?

Dr Rastan. Thank you very much for mentioning this very important issue. This is one of the key questions from all our analyses. We knew from the SYNTAX 1-year data, and this was surprising from the very beginning, that there was a remarkable difference in the 1-year outcome of randomized CABG patients compared with the $\mathrm{CABG}$ registry. We all speculated about the reason for this finding, because randomized patients were obviously healthier and had a less complex coronary anatomy. If anything, the results were expected to be better for randomized patients, not worse. Identifying the reasons for this unexpected finding in surgical patients was one of the major reasons that we made this analysis. 
Of note, we found that the difference between randomized and registry patients was more remarkable in low SYNTAX scores than in higher SYNTAX scores. The reasons were not the randomization itself but the incompleteness of revascularization and the higher rate of repeat revascularization. The following question is, why did randomized patients, or patients with a low SYNTAX score, receive incomplete revascularization more often? This is really interesting but difficult to explain. Thus, we have to analyze this issue further. Maybe it is a matter of the lesion-driven definition of completeness of revascularization that was used in this study.

Just looking at the territories of the LAD, circumflex, and right coronary arteries, we found that there were no significant differences. Thus, it might be possible that the second vessel of a particular territory could make the difference regarding a potential target vessel for the interventionalist to make a PCI postoperatively. The next step is to go more into detail regarding this issue and, more important, to correlate these finding to the clinical symptoms of the patient.

Dr John D. Puskas (Atlanta, $G a$ ). I was particularly fascinated to see that the SYNTAX score did not affect MACCE at 2 years in surgical patients but did in PCI patients. As you know, there are have been several criticisms of database analyses comparing various techniques for CABG surgery on the basis that the Society of
Thoracic Surgeons database, for example, does not have detailed data on coronary anatomy. For instance, there have been those who criticize comparisons of off-pump versus on-pump surgery within the Society of Thoracic Surgeons database because coronary complexity cannot be adjusted for; you have now shown that coronary complexity may in fact not affect MACCE at 2 years when surgery is performed well. I think that is an important point. The inability to adjust for coronary anatomic complexity does not invalidate database comparisons of alternative surgical techniques for CABG.

Dr Rastan. Thank you for this comment. I completely agree that it is actually difficult to quantify the complexity of coronary artery disease. The SYNTAX score is a new tool that we have available. At the moment, it is speculative whether or not the SYNTAX score is really the only true or best instrument to quantify complexity of coronary artery disease. However, it is complex to build and there is a significant interobserver and intraobserver variability. Maybe we should look more at the predictive value of individual components of the SYNTAX score, such as chronic total occlusions or the number of diseased lesions, which also did not affect the surgical results, but might also be predictive for PCI results. However, we as surgeons should also try to better quantify the complexity of artery disease. I agree.

Dr Puskas. Congratulations on a very thought-provoking study. 